\title{
Mass flight activity of Lyonetia prunifoliella malinella (Lepidoptera: Lyonetiidae) with special reference to mating and dispersal
}

\author{
Norio Sekita ${ }^{1}$ \\ Division of Plant Protection and Nutrition, Aomori Apple Experiment Station, Kuroishi, Aomori 036-0332, Japan
}

(Received 7 January 2002; Accepted 31 May 2002)

\begin{abstract}
During observations from September to November, flight activity of Lyonetia prunifoliella malinella occurred in the morning and in the evening outside the crowns of apple and shelter trees. The morning flight started about half an hour before sunrise. About $15 \mathrm{~min}$ later, the number of flying moths increased rapidly and swarms formed. The swarms lasted for about half an hour. Although females were present in the tree crown, they did not participate in the swarms, which were composed only of males. Mating began during the time when the males were swarming, and most of the mating pairs separated by noon. The evening flight started around sunset. It did not develop into swarms and finished in about half an hour. The evening flights were composed of both males and females, with the latter including both virgin and fertilized females. During the evening flight, it was noted that some moths were flying out of view. Simultaneously it was found that the moths flying into view alighted on the shelter trees. Thus, flight in the morning was considered to be associated with mating, while that in the evening with dispersal.
\end{abstract}

Key words: Lyonetia prunifoliella malinella, apple pest, mass flight activity, mating, dispersal

\section{INTRODUCTION}

Two species of leafminer are commonly found in apple orchards in Aomori Prefecture, the northern tip of Honshu, Japan. They are Phyllonorycter ringoniella Matsumura and Lyonetia prunifoliella malinella Matsumura. The former has four generations a year and passes the winter as pupae inside mines in fallen leaves. The latter has five or six generations depending upon the cumulative temperatures in a year. It displays a seasonal dimorphism, with summer and autumn forms. The moths of the summer generations have silvery forewings with black and golden stripes on the rear part, while those of the autumn form, which become the overwintering generation, have grayish forewings with black stripes. Moths of the autumn form mate before hibernation. Males, which lack fat body, die before winter. Only reproductively diapausing females, which have fat bodies and undeveloped ovaries, survive the winter in sheltered places such as a wooden shed within or near orchards (Sekita and Yamada, 1979; Sekita, 1991).

The previous study (Sekita, 2002) explored the association of mass flight activity with mating in $P$. ringoniella. During observations in that study, moths of L. p. malinella were also found to swarm in the apple orchard. In addition, they were found swarming outside the crowns of shelter trees near an apple orchard. Accordingly, observations were conducted for this species in parallel with those on $P$. ringoniella to explore the biological significance of mass flight activity.

\section{MATERIALS AND METHODS}

Study site. The study orchard, located on a hillside in Kashiwagiyama, Kuroishi City, was the same as that used for $P$. ringoniella (cf. Sekita, 2002), and was situated west of an apple orchard belonging to the Aomori Apple Experiment Station (AAES). The shelter trees (areas along D in Fig. 1) had been established along both sides of a road above a steep slope that separated the orchard of AAES into two blocks ( $\mathrm{C}$ and $\mathrm{F}$ in Fig. 1). Along the upper side of the road, nine Japanese alders were planted in a line from west to east. Their height was less than $2 \mathrm{~m}$, because of heavy pruning each spring. The shelter along the lower side of the road was composed of four Japanese alders, two gingkoes, two Japanese hill cherries, two walnuts, four Japanese larches, three Japanese red pines,

\footnotetext{
${ }^{1}$ Present address: Aomori Agricultural Experiment Station, Kuroishi, Aomori 036-0389, Japan
} 
and two Japanese cedars. They formed a line from west to east in the above order. Tree size varied according to the species because no pruning had been carried out. Flight observations were made mainly on Japanese alders on both sides of the road.

Flight observation. Early each morning from 18 September to 18 November 2000, the periphery of the crowns of the shelter trees was surveyed to observe moth activity. The observations generally started before flight activity had begun, and continued until all flight activity finished.

Each evening from 25 September to 17 November excluding eight days $(7,8,13,17$ and 22 October, and 8, 11 and 15 November), observations were made in the same way as was done in the morning. The moths that alighted on the leaves were observed using a flashlight equipped with a red filter.

Weather and sky conditions were recorded each morning and evening at the time of observation. Temperatures were read using a thermometer covered with a thick black-paper envelope and placed within the crown of a shelter tree.

Responses of the swarms to female moths. A tubular metal cage $5.5 \mathrm{~cm}$ long and $7 \mathrm{~cm}$ in diameter with both ends closed with finely meshed net was used to confine living moths. A cage baited with moths was hung inside the tree crown to test the response of the swarm to them. Four cages were hung with about $5 \mathrm{~m}$ separating adjacent cages. The height varied from $20-30 \mathrm{~cm}$ to about $1.5 \mathrm{~m}$ above the ground according to the height of the trees. Moths used in these tests had been reared from the pupal stage. Pupae in cocoons that had been spun at the lower surface of leaves were kept in an insect screen $20 \mathrm{~cm}$ long by $25 \mathrm{~cm}$ wide and $27 \mathrm{~cm}$ high. The leaves were collected on 20 and 22 September from the upper and lower blocks of the apple orchard, AAES (C and F in Fig. 1).

Response measurements were started before the beginning of flight activity each morning and continued until activity ceased. Responses were scored according to the number of moths as devised for $P$. ringoniella (Sekita, 2002); i.e., $0=$ no moths, $1=\mathrm{a}$ few, $2=$ over a few but less than $10,3=$ over 10 but less than 100, and $4=$ over 100 .

All the moths used as bait were dissected later to determine sex and mating status.

Time of mating. To specify the time of mating, moths present among branches as well as on trunks of apple and shelter trees were sought out at various times of day. Moths in copulation were recorded and each of the sites at which copulation was recorded was marked with a pin. During the dark, a flashlight with a red filter was used.

Sex ratio and mating status of flying moths. Some moths that were flying in swarms outside the crowns of apple trees (A in Fig. 1) and shelter trees were captured using a net a few times each week. In addition, moths were caught that were flying

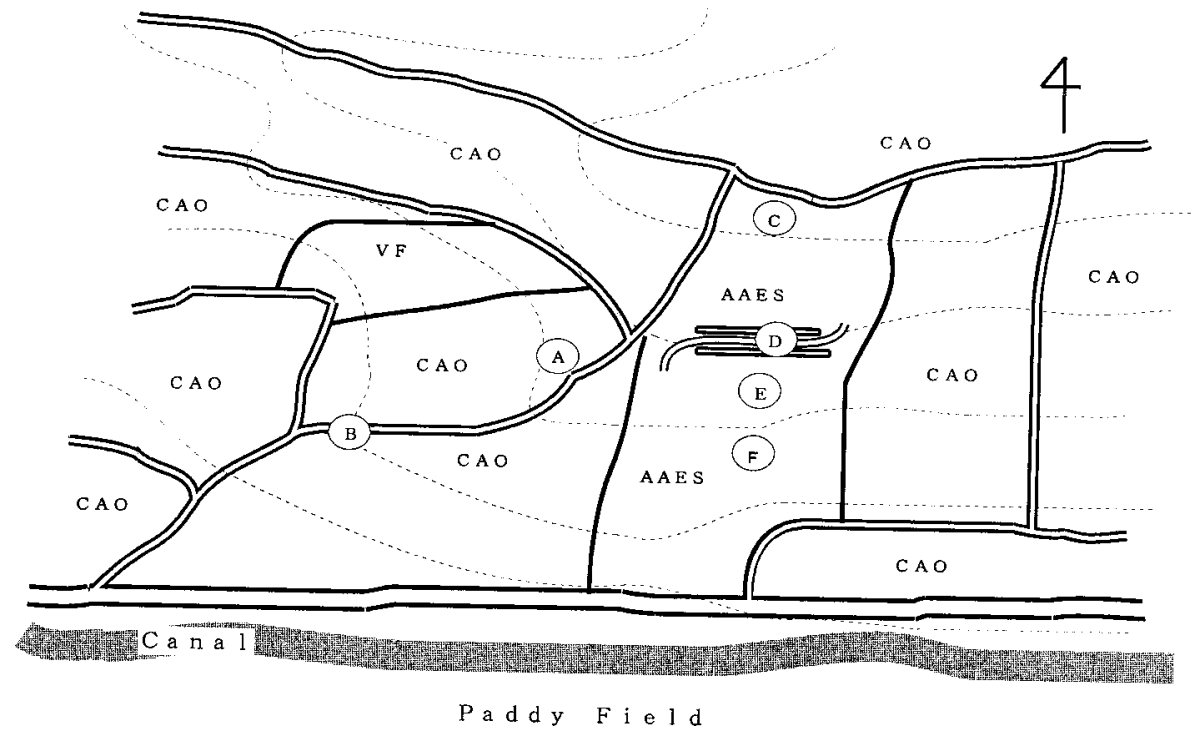

Fig. 1. Map showing the study orchards and shelter trees. $C A O=$ commercial apple orchard, $\mathrm{VF}=$ vegetable field, and AAES = Aomori Apple Experiment Station. Circled letter stands for a site for observation or collection of materials (see text for detail). 
over the road passing through the apple orchards (B in Fig. 1) or over an open space between the shelter trees and the lower block of the apple orchard, AAES (E in Fig. 1). Captured moths were immersed in dilute soapy water to facilitate dissection. After the seasonal morph was recorded, the moths were dissected under a binocular microscope to determine sex. Females were further examined to check the spermatheca to determine whether they had mated or not.

Sex ratio and mating status of moths among branches. To sample moths that were present on the foliage of apple and shelter trees, a small branch (ca. $1 \mathrm{~m}$ long) was pushed into a net and violently shaken. Moths were collected as they took flight on being disturbed. Collections were made not only when the majority of moths were still flying in the swarm, but also at other times of day when no moths were flying. Captured moths were examined in the manner as described above.

\section{RESULTS}

\section{Flight activity in the morning}

Although the density of L. p. malinella was not directly measured during the present study, no leaves on the secondary growth shoots were left without leaf mines in surrounding orchards from late September onward. Data for flight observations are summarized in Table 1 together with the time of sunrise at Aomori City, ca. $20 \mathrm{~km}$ north of the observation site, and sky and weather conditions.

During the 2-month period of observation from 18 September to 18 November, the time of sunrise was delayed $67 \mathrm{~min}$ from 05:20 to 06:27. When the morning just dawned, before sunrise, moths moved onto the upper surface of the leaves from the lower surface, and also from the stems and branches. They walked around the surfaces, actively moving their antennae. The time when flight activity started $(N=37$, range $=04: 42-06: 10)$ was around half an hour before sunrise $(N=37$, mean time $\operatorname{lag} \pm \mathrm{SE}=31 \pm 1 \mathrm{~min}$, range $=5-47 \mathrm{~min})$. At the beginning of flight activity, only a few moths flew sporadically near the surface of the tree crown. About 15 min later $(N=25$, mean $\pm \mathrm{SE}=15 \pm 1 \mathrm{~min}$, range $=5-47 \mathrm{~min})$, the number of flying moths increased, and they formed a swarm. The swarm occurred on the leeward side of shelter trees, and the moths flew in a wave-like movement with both vertical and horizontal components, while facing towards the trees. Each moth used only a restricted space some $300 \mathrm{~cm}$ wide and $100 \mathrm{~cm}$ high within the swarm. When the wind was strong, the moths were trying to head upwind towards the tree crown, but they were forced downwind towards open space or the next tree. When such wind became weaker or stopped, the swarm moved towards the tree crown from which they had been forced out.

The swarming activity continued for about half an hour $(N=33$, mean duration $\pm \mathrm{SE}=25 \pm 2 \mathrm{~min}$, range $=5-60 \mathrm{~min})$. Swarming finished after sunrise, excepting for seven cases finished before sunrise. As the number of flying moths decreased, the number of moths alighting on the upper surface of the leaves increased. With the complete termination of flight activity $(N=43$, range $=05: 55-07: 05)$, all moths disappeared from the upper surface of the leaves. The resting moths were motionless, keeping their antennae backwards along their bodies.

During swarming activity, especially when the wind was moderate, flying moths frequently deviated out of their flight course with a quick turn. These moths rushed at other moths as if they had a flying territory to defend. The moth that was "attacked" shifted its course, and the "attacker" returned quickly to the former flight course.

Among 62 days of morning observations, no flights were recorded on the two days when the morning temperature was equal to or below $0^{\circ} \mathrm{C}$. For the 15 days when the temperature was 1 to $3^{\circ} \mathrm{C}$, flight was recorded on eight occasions, but it did not develop into a swarm. For the 43 days when the temperature was from 4 to $15^{\circ} \mathrm{C}$, flight occurred each day except on one occasion (18 October) when it was gusting; swarming developed on 31 days, but not on the remaining 11 days. A $\chi^{2}$ test showed that flight tended to develop into a swarm under cloudy or rainy conditions but not under clear conditions $(0.025<p<0.05)$. Both flights on two days when the temperature was over $16^{\circ} \mathrm{C}$ developed into swarms.

At $05: 25$ and $05: 50$ on 3 October, $05: 02$ on 5 October, $05: 35$ on 10 October, $05: 45$ on 15 October, 05:32 on 16 October and 05:46 on 19 October, a compact swarm composed of 20-30 moths was seen flying with a repeated upward-and-downward or forward-and-backward movement in a restricted space close to a small branch of less than $1 \mathrm{~m}$ in 
Table 1. Daily observation of morning flight activity of L. p. malinella with time of sunrise and weather conditions

\begin{tabular}{|c|c|c|c|c|c|c|c|c|}
\hline \multirow{2}{*}{ Date } & \multirow{2}{*}{ Sunrise } & \multicolumn{3}{|c|}{ Period } & \multirow{2}{*}{ S.N. ${ }^{a}$} & \multirow{2}{*}{$\begin{array}{c}\text { Temp } \\
{ }^{\circ} \mathrm{C}\end{array}$} & \multirow{2}{*}{ Wind $^{b}$} & \multirow{2}{*}{$\mathrm{Sky}^{\mathrm{c}}$} \\
\hline & & Observation & Flight & Swarm & & & & \\
\hline 18 Sep. & $05: 20$ & $05: 30-06: 20$ & $?^{\mathrm{d}}-06: 10$ & $05: 30-05: 50$ & & 18 & W & $\mathrm{O}$ \\
\hline 19 Sep. & $05: 21$ & $04: 46-06: 20$ & $?-06: 15$ & $05: 05-06: 05$ & & 13 & $\mathrm{~S}$ & $\mathrm{C}$ \\
\hline 20 Sep. & $05: 22$ & $04: 21-06: 00$ & $04: 42-05: 50$ & $04: 55-05: 45$ & & 12 & LW & $\mathrm{C}$ \\
\hline 21 Sep. & $05: 23$ & $04: 55-06: 15$ & $?-06: 05$ & No swarm & & 11 & LW & $\mathrm{C}$ \\
\hline 22 Sep. & $05: 24$ & $04: 45-06: 15$ & $?-06: 05$ & $05: 00-05: 45$ & & 12 & LW & $\mathrm{O}$ \\
\hline 23 Sep. & $05: 25$ & $04: 50-06: 50$ & $05: 00-06: 15$ & $05: 25-06: 05$ & & 13 & $\mathrm{~S}$ & $\mathrm{O}$ \\
\hline 24 Sep. & $05: 26$ & $04: 35-06: 25$ & $05: 00-06: 16$ & $05: 25-06: 05$ & & 14 & $\mathrm{~S}$ & LR \\
\hline 25 Sep. & $05: 27$ & $04: 40-06: 06$ & $04: 50-05: 50$ & $05: 15-05: 45$ & & 16 & LW & $\mathrm{C}$ \\
\hline 26 Sep. & $05: 28$ & $04: 30-06: 00$ & $05: 01-05: 50$ & No swarm & & 14 & LW & $\mathrm{C}$ \\
\hline 27 Sep. & $05: 29$ & $04: 50-06: 20$ & $04: 55-05: 50$ & $05: 22-05: 40$ & & 11 & $\mathrm{~S}$ & $\mathrm{R}$ \\
\hline 28 Sep. & $05: 30$ & $05: 07-05: 55$ & $?-05: 55$ & No swarm & & 12 & $\mathrm{~S}$ & $\mathrm{O}$ \\
\hline 29 Sep. & $05: 31$ & 04:45-06:09 & 04:47-06:09 & $05: 05-05: 50$ & & 11 & LW & $\mathrm{O}$ \\
\hline 30 Sep. & $05: 32$ & $04: 50-06: 22$ & $05: 00-06: 02$ & $05: 12-05: 41$ & & 11 & LW & $\mathrm{C}$ \\
\hline 1 Oct. & $05: 33$ & $05: 20-06: 05$ & $?-06: 05$ & No swarm & & 13 & $\mathrm{~S}$ & $\mathrm{C}$ \\
\hline 2 Oct. & $05: 34$ & $05: 05-05: 45$ & $05: 20-?^{\mathrm{e}}$ & $05: 20-05: 45$ & & 11 & $\mathrm{~S}$ & $\mathrm{O}$ \\
\hline 3 Oct. & $05: 35$ & $04: 55-06: 05$ & $05: 05-06: 05$ & $05: 20-05: 50$ & O & 14 & $\mathrm{~S}$ & $\mathrm{R}$ \\
\hline 4 Oct. & $05: 36$ & $05: 50-06: 30$ & ?-06:28 & No swarm & & 11 & $\mathrm{~S}$ & $\mathrm{C}$ \\
\hline 5 Oct. & $05: 37$ & $04: 40-05: 55$ & $04: 50-05: 50$ & $05: 00-05: 05$ & O & 8 & LW & $\mathrm{C}$ \\
\hline 6 Oct. & $05: 38$ & $05: 10-06: 15$ & $?-06: 10$ & $05: 20-05: 45$ & & 7 & LW & $\mathrm{C}$ \\
\hline 7 Oct. & $05: 39$ & $04: 35-06: 25$ & $04: 58-06: 07$ & $05: 05-05: 40$ & & 7 & $\mathrm{~W}$ & $\mathrm{R}$ \\
\hline 8 Oct. & $05: 40$ & $05: 20-06: 36$ & $?-06: 35$ & No swarm & & 9 & LW & $\mathrm{O}$ \\
\hline 9 Oct. & $05: 41$ & $04: 45-06: 28$ & $05: 04-06: 10$ & $05: 10-05: 37$ & & 12 & LW & $\mathrm{O}$ \\
\hline 10 Oct. & $05: 42$ & $04: 35-06: 21$ & $05: 05-06: 15$ & $05: 15-05: 57$ & O & 10 & $\mathrm{~S}$ & $\mathrm{O}$ \\
\hline 11 Oct. & $05: 43$ & $04: 32-06: 20$ & $05: 10-06: 18$ & $05: 31-05: 57$ & & 7 & LW & $\mathrm{O}$ \\
\hline 12 Oct. & $05: 44$ & $04: 50-06: 20$ & $05: 06-06: 20$ & $05: 30-06: 02$ & & 11 & LW & $\mathrm{M}$ \\
\hline 13 Oct. & $05: 45$ & $05: 10-06: 27$ & $?-06: 25$ & $05: 40-05: 45$ & & 5 & LW & $\mathrm{O}$ \\
\hline 14 Oct. & $05: 46$ & $04: 52-06: 15$ & $05: 20-06: 12$ & $05: 25-05: 32$ & & 6 & $\mathrm{~S}$ & $\mathrm{O}$ \\
\hline 15 Oct. & $05: 47$ & $05: 15-06: 15$ & $05: 17-06: 11$ & $05: 40-05: 46$ & O & 4 & $\mathrm{~S}$ & $\mathrm{O}$ \\
\hline 16 Oct. & $05: 48$ & $04: 57-06: 50$ & $05: 20-06: 48$ & $05: 25-05: 50$ & O & 7 & $\mathrm{~S}$ & $\mathrm{O}$ \\
\hline 17 Oct. & $05: 50$ & $05: 03-06: 36$ & $05: 40-06: 35$ & No swarm & & 1 & LW & $\mathrm{C}$ \\
\hline 18 Oct. & $05: 51$ & $05: 45-07: 50$ & No flight & & & 5 & $\mathrm{G}$ & LR \\
\hline 19 Oct. & $05: 52$ & $05: 00-07: 15$ & $05: 21-07: 05$ & No swarm & O & 4 & $\mathrm{~S}$ & $\mathrm{O}$ \\
\hline 20 Oct. & $05: 53$ & $05: 20-07: 00$ & $05: 30-06: 40$ & No swarm & & 3 & $\mathrm{~S}$ & $\mathrm{C}$ \\
\hline 21 Oct. & $05: 54$ & $05: 07-06: 05$ & $05: 15-06: 00$ & $05: 26-05: 57$ & & 11 & $\mathrm{~S}$ & $\mathrm{O}$ \\
\hline 22 Oct. & $05: 55$ & $05: 35-06: 45$ & $?-06: 45$ & $06: 10-06: 25$ & & 7 & $\mathrm{~S}$ & $\mathrm{C}$ \\
\hline 23 Oct. & $05: 56$ & $05: 02-06: 30$ & $05: 15-?$ & No swarm & & 3 & LW & $\mathrm{C}$ \\
\hline 24 Oct. & $05: 57$ & $05: 00-06: 30$ & $05: 15-?$ & No swarm & $\triangle$ & 3 & LW & $\mathrm{C}$ \\
\hline 25 Oct. & $05: 58$ & $05: 08-06: 31$ & $05: 18-?$ & $05: 40-06: 07$ & $\triangle$ & 12 & LW & $\mathrm{O}$ \\
\hline 26 Oct. & $05: 59$ & $05: 05-06: 46$ & $?-06: 44$ & $05: 40-06: 10$ & $\triangle$ & 8 & $\mathrm{~S}$ & $\mathrm{O}$ \\
\hline 27 Oct. & 06:01 & $05: 15-06: 40$ & $05: 25-06: 40$ & No swarm & $\triangle$ & 4 & $\mathrm{~S}$ & $\mathrm{O}$ \\
\hline 28 Oct. & 06:02 & $05: 00-07: 15$ & $05: 17-?$ & No swarm & & 3 & $\mathrm{~S}$ & $\mathrm{O}$ \\
\hline 29 Oct. & 06:03 & $05: 15-06: 45$ & $05: 32-06: 45$ & $05: 40-05: 50$ & $\triangle$ & 6 & $\mathrm{~S}$ & $\mathrm{O}$ \\
\hline 30 Oct. & 06:04 & $05: 05-06: 30$ & $05: 45-06: 30$ & $06: 10-06: 25$ & $\triangle$ & 4 & $\mathrm{~S}$ & $\mathrm{R}$ \\
\hline 31 Oct. & 06:05 & $05: 10-06: 45$ & $05: 34-06: 45$ & No swarm & & 2 & LW & $\mathrm{C}$ \\
\hline 1 Nov. & 06:07 & $05: 13-06: 15$ & 05:36-? & 05:50-06:00 & & 8 & LW & $\mathrm{O}$ \\
\hline 2 Nov. & 06:08 & $05: 20-06: 05$ & $05: 40-?$ & $06: 00-06: 05$ & & 7 & $\mathrm{~S}$ & $\mathrm{R}$ \\
\hline 3 Nov. & 06:09 & $06: 10-07: 00$ & $?-06: 45$ & No swarm & & 6 & LW & $\mathrm{C}$ \\
\hline 4 Nov. & $06: 10$ & $06: 20-06: 55$ & No flight & & & 3 & $\mathrm{~W}$ & $\mathrm{C}$ \\
\hline 5 Nov. & $06: 11$ & $05: 50-06: 55$ & $?-06: 55$ & $06: 00-06: 15$ & & 9 & $\mathrm{G}$ & $\mathrm{O}$ \\
\hline 6 Nov. & $06: 13$ & $05: 28-06: 50$ & $05: 45-06: 35$ & No swarm & & 2 & LW & $\mathrm{C}$ \\
\hline 7 Nov. & $06: 14$ & $05: 30-06: 45$ & $05: 45-06: 15$ & No swarm & $\triangle$ & 6 & LW & $\mathrm{C}$ \\
\hline 8 Nov. & $06: 15$ & $05: 55-07: 00$ & $06: 10-?$ & $06: 18-06: 40$ & & 4 & LW & $\mathrm{O}$ \\
\hline
\end{tabular}


Table 1. (Continued)

\begin{tabular}{|c|c|c|c|c|c|c|c|c|}
\hline \multirow{2}{*}{ Date } & \multirow{2}{*}{ Sunrise } & \multicolumn{3}{|c|}{ Period } & \multirow{2}{*}{ S.N. ${ }^{a}$} & \multirow{2}{*}{$\begin{array}{c}\text { Temp } \\
{ }^{\circ} \mathrm{C}\end{array}$} & \multirow{2}{*}{ Wind $^{\mathrm{b}}$} & \multirow{2}{*}{$\mathrm{Sky}^{\mathrm{c}}$} \\
\hline & & Observation & Flight & Swarm & & & & \\
\hline 9 Nov. & $06: 16$ & $06: 00-06: 35$ & No flight & & & 2 & S & $\mathrm{O}$ \\
\hline 10 Nov. & $06: 17$ & $06: 00-06: 55$ & $06: 05-?$ & No swarm & & 2 & $\mathrm{~S}$ & LR \\
\hline $11 \mathrm{Nov}$. & 06:19 & $06: 00-06: 35$ & No flight & & & 1 & LW & $\mathrm{S}$ \\
\hline 12 Nov. & $06: 20$ & $06: 45-07: 00$ & No flight & & & 1 & LW & $\mathrm{O}$ \\
\hline 13 Nov. & $06: 21$ & $05: 55-06: 25$ & No flight & & & 2 & $\mathrm{~W}$ & LR \\
\hline 14 Nov. & $06: 22$ & $07: 00-07: 05$ & No flight & & & 3 & LW & $\mathrm{O}$ \\
\hline 15 Nov. & $06: 23$ & $06: 25-07: 05$ & $?-07: 00$ & No swarm & & 9 & $\mathrm{~S}$ & $\mathrm{O}$ \\
\hline 16 Nov. & $06: 25$ & $06: 30-06: 40$ & No flight & & & 0 & LW & $\mathrm{O}$ \\
\hline 17 Nov. & $06: 26$ & $06: 30-06: 40$ & No flight & & & -1 & LW & $\mathrm{O}$ \\
\hline 18 Nov. & $06: 27$ & $06: 20-07: 05$ & No flight & & & 2 & $\mathrm{~S}$ & $\mathrm{R}$ \\
\hline
\end{tabular}

${ }^{\text {a }}$ Special note: $\bigcirc$ indicates the date when a large number of moths flew close to a small branch, and $\Delta$ the date when moths crawled down along a branch (see text for detail).

${ }^{\mathrm{b}}$ Wind: $\mathrm{S}=$ still, $\mathrm{LW}=$ light wind, $\mathrm{W}=$ windy, and $\mathrm{G}=$ gusting.

${ }^{\mathrm{c}} \mathrm{Sky}$ : $\mathrm{C}=$ clear, $\mathrm{O}=$ overcast, $\mathrm{M}=$ mist, $\mathrm{LR}=$ light rain, $\mathrm{R}=$ rain, and $\mathrm{S}=$ snow.

${ }^{\mathrm{d}}$ Flight had started before observation.

${ }^{\mathrm{e}}$ Flight had not terminated when observations ended.

length (the dates are marked with $\bigcirc$ in the column of special comment of Table 1). The pattern of movement differed markedly from that of swarms which flew facing towards the trees. Thus, the swarm close to the small branch was compact and flew actively in a repeated movement with either a vertical or horizontal component in a small space, whereas the swarm which flew facing the trees was widely spread out and flew slowly in a repeated wave-like movement with both vertical and horizontal components over a relatively wide space. These compact swarms close to the small branch usually occurred during the period when the widespread swarms facing towards the trees occurred, and suddenly disappeared after a few minutes. It was evident that these swarms were in response to females, since a calling female was present on the branch in the first two cases (no searches were made in other cases).

On 24 October it was cold $\left(3^{\circ} \mathrm{C}\right)$ and clear. Although some flights occurred, a swarm did not develop. At 05:44 that morning, moths were noticed crawling down sluggishly towards the trunk of a shelter tree forming lines along a branch. After a while they formed a crowd around each of four moths that had been present near a crotch where the branch met the main trunk. Before long, a mating pair was established with each of these four groups of moths. A similar phenomenon was found on 25, 26, 27, 29 and 30 October, and 7 November (marked with $\triangle$ in Table 1), and one mating pair was established on 29 October. Except for 25 October when it was mild $\left(12^{\circ} \mathrm{C}\right)$, all mornings were cold (mean temperature $=5.6^{\circ} \mathrm{C}$, range $=4-8^{\circ} \mathrm{C}$ ) and no swarms developed on 27 October and 7 November.

\section{Flight in the evening}

Data for flight observations in the evening are summarized in Table 2, together with the time of sunset, and sky and weather conditions. During the 54 days of observation, from 25 September to 17 November, the time of sunset advanced $67 \mathrm{~min}$ from 17:29 to 16:22. Before any flight activity, moths appeared on the upper surface of the leaves, moving from the lower surface or from the stems and branches. They walked around the surface actively moving their antennae. The time when flight activity started $(N=27$, range $=15: 52-17: 52)$ was around the time of sunset $(N=27$, mean time $\operatorname{lag} \pm \mathrm{SE}=0 \pm 3 \mathrm{~min}$, range $=-43-48 \mathrm{~min})$. In contrast to morning flights, evening flights did not develop into a swarm and remained sporadic, continuing for about half an hour $(N=22$, mean $\pm \mathrm{SE}=$ $26 \pm 4 \mathrm{~min}$, range $=5-73 \mathrm{~min})$. As the number of moths flying decreased, the number that alighted on the upper surface of leaves increased. With the complete finish of flight activity, all the moths 
Table 2. Daily observation of evening flight activity of L. p. malinella with time of sunset and weather conditions

\begin{tabular}{|c|c|c|c|c|c|c|}
\hline \multirow{2}{*}{ Date } & \multirow{2}{*}{ Sunset } & \multicolumn{2}{|c|}{ Period } & \multirow{2}{*}{$\begin{array}{c}\text { Temp. } \\
{ }^{\circ} \mathrm{C}\end{array}$} & \multirow{2}{*}{ Wind } & \multirow{2}{*}{ Sky } \\
\hline & & Observation & Flight & & & \\
\hline 25 Sep. & $17: 29$ & $17: 40-17: 50$ & $17: 40-17: 50$ & 17 & $\mathrm{~S}$ & $\mathrm{C}$ \\
\hline 26 Sep. & $17: 27$ & $16: 50-17: 45$ & $17: 00-17: 10$ & 14 & LW & $\mathrm{C}$ \\
\hline 27 Sep. & $17: 26$ & $16: 55-17: 50$ & $17: 30-17: 48$ & 13 & $\mathrm{~S}$ & $\mathrm{R}$ \\
\hline 28 Sep. & $17: 24$ & $16: 32-18: 08$ & $16: 45-17: 46$ & 17 & LW & $\mathrm{C}$ \\
\hline 29 Sep. & $17: 22$ & $17: 20-18: 10$ & $17: 30-17: 45$ & 17 & $\mathrm{~S}$ & $\mathrm{O}$ \\
\hline 30 Sep. & $17: 21$ & $17: 05-18: 00$ & $17: 30-17: 40$ & 19 & $\mathrm{~S}$ & $\mathrm{O}$ \\
\hline 1 Oct. & $17: 19$ & $16: 25-18: 05$ & $17: 20-17: 50$ & 21 & $\mathrm{~S}$ & $\mathrm{C}$ \\
\hline 2 Oct. & $17: 18$ & $17: 05-17: 55$ & $17: 20-17: 45$ & 18 & $\mathrm{~S}$ & $\mathrm{O}$ \\
\hline 3 Oct. & $17: 16$ & $17: 07-17: 50$ & $17: 35-17: 40$ & 17 & LW & $\mathrm{C}$ \\
\hline 4 Oct. & $17: 14$ & $17: 00-18: 04$ & $17: 32-17: 40$ & 15 & LW & $\mathrm{O}$ \\
\hline 5 Oct. & $17: 13$ & $16: 55-17: 45$ & $?-17: 40$ & 12 & W & $\mathrm{R}$ \\
\hline 6 Oct. & $17: 11$ & $17: 00-17: 45$ & $17: 10-17: 40$ & $?$ & $\mathrm{~S}$ & $\mathrm{O}$ \\
\hline 7 Oct. & $17: 10$ & No observation & & & & \\
\hline 8 Oct. & $17: 08$ & No observation & & & & \\
\hline 9 Oct. & $17: 06$ & $17: 05-17: 40$ & $17: 15-17: 35$ & 17 & LW & $\mathrm{O}$ \\
\hline 10 Oct. & $17: 05$ & $16: 55-17: 40$ & $17: 13-17: 35$ & 14 & $\mathrm{~S}$ & $\mathrm{O}$ \\
\hline 11 Oct. & $17: 03$ & $17: 20-18: 07$ & $17: 52-?$ & 17 & G & $\mathrm{O}$ \\
\hline 12 Oct. & $17: 02$ & $17: 20-17: 55$ & No flight & 9 & W & $\mathrm{O}$ \\
\hline 13 Oct. & $17: 00$ & No observation & & & & \\
\hline 14 Oct. & $16: 58$ & $17: 28-17: 40$ & No flight & 10 & $\mathrm{~S}$ & $\mathrm{O}$ \\
\hline 15 Oct. & $16: 57$ & $17: 28-17: 38$ & No flight & 11 & $\mathrm{~S}$ & $\mathrm{R}$ \\
\hline 16 Oct. & $16: 55$ & $17: 00-17: 40$ & $17: 04-17: 35$ & 9 & LW & $\mathrm{C}$ \\
\hline 17 Oct. & $16: 54$ & No observation & & & & \\
\hline 18 Oct. & $16: 52$ & $16: 37-17: 10$ & No flight & 4 & $\mathrm{~S}$ & $\mathrm{O}$ \\
\hline 19 Oct. & $16: 51$ & $16: 00-17: 15$ & $16: 15-17: 15$ & 13 & $\mathrm{~S}$ & $\mathrm{C}$ \\
\hline 20 Oct. & $16: 49$ & $16: 48-17: 20$ & $16: 52-17: 20$ & 12 & $\mathrm{~S}$ & LR \\
\hline 21 Oct. & $16: 48$ & $16: 47-17: 25$ & No flight & 11 & LW & $\mathrm{C}$ \\
\hline 22 Oct. & $16: 47$ & No observation & & & & \\
\hline 23 Oct. & $16: 45$ & $16: 35-17: 10$ & $?-17: 00$ & 9 & $\mathrm{~S}$ & $\mathrm{O}$ \\
\hline 24 Oct. & $16: 44$ & $16: 44-17: 05$ & $17: 00-?$ & 13 & LW & $\mathrm{O}$ \\
\hline 25 Oct. & $16: 42$ & $16: 40-17: 24$ & No flight & 14 & $\mathrm{~S}$ & RL \\
\hline 26 Oct. & $16: 41$ & $16: 45-17: 28$ & No flight & 5 & LW & LR \\
\hline 27 Oct. & $16: 40$ & $16: 10-17: 05$ & $16: 46-16: 55$ & 6 & $\mathrm{~S}$ & $\mathrm{O}$ \\
\hline 28 Oct. & $16: 38$ & $16: 13-17: 00$ & $16: 30-17: 00$ & 9 & LW & $\mathrm{O}$ \\
\hline 29 Oct. & $16: 37$ & $16: 41-17: 14$ & $16: 50-?$ & 7 & $\mathrm{~S}$ & $\mathrm{C}$ \\
\hline 30 Oct. & $16: 36$ & $16: 25-17: 15$ & No flight & 6 & LW & $\mathrm{O}$ \\
\hline 31 Oct. & $16: 35$ & $15: 50-17: 05$ & $15: 52-17: 05$ & 13 & LW & $\mathrm{C}$ \\
\hline 1 Nov. & $16: 34$ & $16: 10-17: 00$ & $?-16: 55$ & 8 & $\mathrm{~S}$ & LR \\
\hline 2 Nov. & $16: 32$ & $16: 05-16: 35$ & $16: 25-?$ & 7 & $\mathrm{~S}$ & LR \\
\hline 3 Nov. & $16: 31$ & $16: 50-17: 20$ & $?-17: 15$ & 11 & $\mathrm{~S}$ & $\mathrm{C}$ \\
\hline 4 Nov. & $16: 30$ & $16: 08-17: 10$ & $16: 20-17: 10$ & 10 & $\mathrm{~S}$ & $\mathrm{C}$ \\
\hline 5 Nov. & $16: 29$ & $16: 13-17: 00$ & $16: 50-?$ & 12 & G & $\mathrm{O}$ \\
\hline 6 Nov. & $16: 28$ & $16: 10-16: 45$ & $?-16: 40$ & 11 & $\mathrm{~S}$ & $\mathrm{C}$ \\
\hline 7 Nov. & $16: 27$ & $16: 20-17: 00$ & $?-16: 56$ & 16 & W & $\mathrm{O}$ \\
\hline 8 Nov. & $16: 26$ & No observation & & & & \\
\hline 9 Nov. & $16: 25$ & $16: 00-16: 35$ & $?-16: 30$ & 5 & $\mathrm{~S}$ & $\mathrm{C}$ \\
\hline 10 Nov. & $16: 24$ & $16: 00-16: 45$ & $16: 20-16: 40$ & 3 & $\mathrm{~S}$ & $\mathrm{O}$ \\
\hline 11 Nov. & $16: 23$ & No observation & & & & \\
\hline 12 Nov. & $16: 22$ & $16: 00-16: 20$ & $?-16: 15$ & 6 & $\mathrm{~S}$ & $\mathrm{O}$ \\
\hline 13 Nov. & $16: 21$ & $16: 15-16: 30$ & No flight & 4 & $\mathrm{~S}$ & $\mathrm{O}$ \\
\hline 14 Nov. & $16: 20$ & 16:58-? & No flight & 7 & LW & $\mathrm{C}$ \\
\hline 15 Nov. & $16: 19$ & No observation & & & & \\
\hline 16 Nov. & $16: 18$ & $16: 40-16: 45$ & No flight & & & \\
\hline 17 Nov. & $16: 17$ & $16: 15-16: 45$ & $16: 20-16: 35$ & 4 & $\mathrm{~S}$ & $\mathrm{O}$ \\
\hline
\end{tabular}

For explanation of the symbols, see Table 1. 
Table 3. The number of L. p. malinella moths flying away from or into the shelter trees

\begin{tabular}{cccc}
\hline Date & $\begin{array}{c}\text { Time of } \\
\text { observation }\end{array}$ & $\begin{array}{c}\text { No. moths } \\
\text { flying out }\end{array}$ & $\begin{array}{c}\text { No. moths } \\
\text { flying in }\end{array}$ \\
\hline 19 Oct. & $16: 20-17: 15$ & 100 & 63 \\
28 & $16: 37-16: 45$ & 7 & 9 \\
& $16: 55-17: 00$ & 1 & 0 \\
31 & $16: 45-17: 05$ & 10 & 5 \\
1 Nov. & $16: 30-16: 40$ & 7 & 3 \\
4 & $16: 50-17: 00$ & 1 & 0 \\
6 & $16: 38-16: 42$ & 18 & 2 \\
7 & $16: 25-16: 45^{\text {a }}$ & 2 & 66 \\
10 & $16: 42-17: 00$ & 2 & 2 \\
& $16: 30-16: 45$ & 2 & 3 \\
\hline
\end{tabular}

${ }^{a}$ A disturbance occurred in the orchards with harvest.

gradually disappeared from view on the leaf surface.

During these observations, moths that had taken flight out of the shelter trees were frequently observed to fly out of view. Further, moths flying into view and alighting on the shelter trees were observed. Supplementary observations made at an open space (E in Fig. 1) demonstrated that there was considerable movement of moths both into and out of the observed area. For instance, during $55 \mathrm{~min}$ from $16: 20$ to $17: 15$ on 19 October, 100 moths flew out and 63 moths flew into view to alight on the shelter trees (Table 3). On 6 November, the apple trees were disturbed by harvesting, resulting in a marked increase in the number of moths that flew into the shelter trees.

\section{Response of swarms to females}

Table 4 shows the dates when the traps were operated and the maximum response score recorded for each trap during this period. On 22 September, a virgin female of the autumn form with undeveloped ovaries was used as bait in each of four traps. From 04:55 to 06:00 each trap was observed 12 times at about 5-min intervals (data not presented). A maximum response score of 2 was recorded at 05:30 for each of the traps. All moths seen flew on the leeward side of the traps. Although the duration of flights noted was only a few minutes, all of these flights were clearly responses to the female used as bait because the moths observed were hovering with a repeated forward-and-backward movement compactly facing the upwind to the cage. The
Table 4. Responses of L. p. malinella to various baits in the traps

\begin{tabular}{|c|c|c|c|}
\hline $\begin{array}{l}\text { Date trap } \\
\text { operated }\end{array}$ & Trap & Bait used & $\begin{array}{l}\text { Maximum } \\
\text { score }\end{array}$ \\
\hline \multirow[t]{4}{*}{22 Sep. } & $\# 1$ & 1 unmated immature ${ }^{\mathrm{a}} q_{\mathrm{A}}^{\mathrm{b}}$ & 2 \\
\hline & $\# 2$ & 1 unmated immature $q_{\mathrm{A}}$ & 2 \\
\hline & $\# 3$ & 1 unmated immature ${ }_{\mathrm{A}}$ & 2 \\
\hline & $\# 4$ & 1 unmated immature ${ }_{\mathrm{A}}$ & 2 \\
\hline \multirow[t]{4}{*}{ 23-25 Sep. } & $\# 1$ & 1 unmated mature ${ }^{\circ}$ & 1 \\
\hline & $\# 2$ & $1 \hat{\sigma}_{\mathrm{A}}$ & 1 \\
\hline & $\# 3$ & blank & 0 \\
\hline & $\# 4$ & 1 unmated immature ${ }{ }_{\mathrm{A}}$ & 1 \\
\hline \multirow[t]{4}{*}{ 26-28 Sep. } & $\# 1$ & 1 unmated immature $q_{\mathrm{A}}$ & 1 \\
\hline & $\# 2$ & 1 unmated immature $q_{\mathrm{A}}$ & 0 \\
\hline & $\# 3$ & 1 mated mature $q_{\mathrm{A}}$ & 0 \\
\hline & $\# 4$ & 1 unmated mature $q_{S}$ & 1 \\
\hline \multirow[t]{4}{*}{ 6-11 Oct. } & $\# 1$ & 1 unmated immature $q_{\mathrm{A}}$ & 1 \\
\hline & $\# 2$ & $1 \delta_{\mathrm{A}}$ & 0 \\
\hline & $\# 3$ & 1 mated immature $q_{\mathrm{A}}$ & 0 \\
\hline & $\# 4$ & $1 \hat{o}_{\mathrm{A}}$ & 0 \\
\hline \multirow[t]{4}{*}{ 13-20 Oct. } & $\# 1$ & $\begin{array}{l}1 \text { unmated immature } q_{\mathrm{A}}+ \\
1 \text { mated immature }{ }_{q_{\mathrm{A}}}+1 \widehat{\delta}_{\mathrm{A}}\end{array}$ & 4 \\
\hline & $\# 2$ & 1 mated immature ${ }_{A}$ & 1 \\
\hline & \#3 & 1 unmated immature ${ }{ }_{\mathrm{A}}$ & 4 \\
\hline & $\# 4$ & 1 unmated immature ${ }_{\mathrm{A}}$ & 2 \\
\hline \multirow[t]{4}{*}{ 25-27 Oct. } & $\# 1$ & 1 mated immature $q_{\mathrm{A}}$ & 0 \\
\hline & $\# 2$ & blank & 0 \\
\hline & $\# 3$ & 1 unmated immature $q_{\mathrm{A}}$ & 0 \\
\hline & $\# 4$ & blank & 0 \\
\hline
\end{tabular}

${ }^{\text {a }}$ A female moth with undeveloped ovaries was described as immature, and those having ovaries with mature eggs as mature.

b Subscripts "A" and "S" are the autumn and summer forms, respectively.

flight pattern was quite similar to the one in which moths flew in response to females that were calling on small branches. From 13 to 20 October, moths flew frequently each morning to the leeward of trap $\# 1$ and $\# 3$, and the daily maximum scores ranged from 3 to 4 for the former, and 2 to 4 for the latter. However, the observed flight pattern was not compact, and differed from that seen when moths flew in response to females calling on the small branches. In other observations, although flights were sometimes observed near the traps, they were sporadic. It was not possible to determine whether or not the moths were responding to the trap.

Although supplementary observations were made later in the morning and around sunset, no responses to the traps were observed at these times (data not presented). 
Table 5. The percent of L. p. malinella moths that were in pairs in each sample $(p)$ and average percent in all samples $(\hat{p})$ in relation to the time of observation

\begin{tabular}{lccccl}
\hline \multicolumn{1}{c}{ Time } & No. of samples & Range of sample size & S.p. & Range of $p$ & $\hat{p}^{\mathrm{b}}$ \\
\hline Before flight activity & 1 & 16 & 0 & 0 & 0 \\
During flight activity & 38 & $12-155$ & 17 & $0-62.1$ & 4.3 \\
Soon after flight activity & 19 & $24-258$ & 11 & $0-11.8$ & 4.1 \\
Until midday & 3 & $48-82$ & 2 & $0-12.5$ & 4.3 \\
In the afternoon & 10 & $10-293$ & 1 & $0-0.7$ & 0.2 \\
Around sunset & 11 & $14-127$ & 2 & $0-5.0$ & 0.8 \\
\hline
\end{tabular}

${ }^{\mathrm{a}}$ The number of samples in which pairs were present.

${ }^{\mathrm{b}}$ Calculated with both total number of pairs and the total number of individuals summed for all samples.

Table 6. The sex ratio and mating status of flying moths of L. p. malinella

\begin{tabular}{|c|c|c|c|c|c|c|}
\hline Flight time & Site & Date & No. samples & Total moths & $\%$ females & $\%$ mated females \\
\hline \multirow[t]{3}{*}{ Morning } & Apple orchard & 31 Aug.-7 Oct. & 9 & 128 & 0 & - \\
\hline & Shelter trees & 19 Sep.-8 Nov. & 5 & 240 & 0 & - \\
\hline & Road & 13 Oct. -5 Nov. & 4 & 128 & $1.6^{\mathrm{a}}$ & - \\
\hline \multirow{2}{*}{ Evening } & Road & 19 Oct.-3 Nov. & 5 & 55 & 63.4 & 45.7 \\
\hline & Open space & 3-4 Nov. & 2 & 32 & 46.9 & 80.0 \\
\hline
\end{tabular}

${ }^{\text {a }}$ A sample from 5 November contained females because of gusting wind (see text).

\section{The time of mating}

The percent of moths that were in pairs $(p)$ was calculated using the formula $p=100 \times 2 \times n_{\mathrm{P}} / n_{\mathrm{T}}$, where $n_{\mathrm{P}}$ is the number of pairs, and $n_{\mathrm{T}}$ is the total number of females and males. In 82 of 90 samples, the $n_{\mathrm{T}}$ was equal to or over 10 . The results for these 82 samples were grouped for six categories of the times of day, i.e., 1) before flight activity, 2) during flight activity, 3) soon after flight activity (until ca. $08: 30)$, 4) from then until midday, 5) in the afternoon (before sunset), and 6) around sunset (Table 5).

Only one observation was made before flight activity began. At this time, all moths remained motionless, and no mating pairs were found. During the time when flight activity was at its peak, the mean percentage of moths mating was $4.3 \%$. Moths that were not mating were restless, actively moving their antennae, which were held upright and forward. Almost all pairs that were present soon after the flight period were those that had been established during that time. At other times of day, no new pairs were established, and the moths remained motionless until sunset. Table 5 also indicates that almost all pairs separated by the afternoon.

\section{Sex ratios and the mating status of flying moths}

The number of moths captured from swarms and the proportion of females are shown in Table 6, together with the mating status of the female moths. All moths captured in the morning were males, except for a sample on 5 November when two out of 13 moths were females. However, these data were clearly exceptional because it was gusting that day and the branches were shaking violently enough to cause the moths to take flight.

In contrast, both males and females were always present in evening samples regardless of the date and site of collection. Females included mated and unmated moths.

\section{Sex ratios and the mating status of moths among branches}

The number of moths collected from branches and the proportion of females are shown in Table 7 , together with the proportion of females that had mated. Irrespective of the trees and times of collection, females were present in all samples of moths from branches with only three exceptions. These were collected during flight activity from shelter trees. The reason why the females were absent from these samples is not known. The females 
Table 7. The sex ratio and mating status of L. p. malinella moths present on branches

\begin{tabular}{|c|c|c|c|c|c|c|}
\hline Tree & Time of day & Date & No. samples & Total moths & $\%$ females & $\%$ mated females \\
\hline \multirow[t]{4}{*}{ Apple } & During flight activity & 8 Sep. -7 Oct. & 9 & 338 & 32.2 & 46.8 \\
\hline & Soon after flight activity & 15-17 Sep. & 2 & 89 & 46.1 & 87.8 \\
\hline & Midday & 31 Aug. & 1 & 5 & 40.0 & 100.0 \\
\hline & Around sunset & 19 Sep. & 1 & 45 & 28.9 & 46.2 \\
\hline \multirow[t]{4}{*}{ Shelter } & During flight activity & 21 Sep.-26 Oct. & $6^{\mathrm{a}}$ & 325 & 10.2 & 33.3 \\
\hline & Soon after flight activity & 18 Sep.-7 Oct. & 3 & 351 & 22.2 & 55.1 \\
\hline & Midday & 29 Sep.-16 Nov. & 3 & 136 & 22.1 & 56.7 \\
\hline & Around sunset & 19 Sep. & 1 & 247 & 4.5 & 54.6 \\
\hline
\end{tabular}

${ }^{\text {a }}$ Three of six samples had no females (see text).

Table 8. Proportions of L. p. malinella females that were present as the autumn form and those that were immature

\begin{tabular}{lrcc}
\hline \multicolumn{1}{c}{ Date } & $\begin{array}{c}\text { No. } \\
\text { females }\end{array}$ & $\begin{array}{c}\text { \% autumn } \\
\text { form }\end{array}$ & $\begin{array}{c}\% \\
\text { immature }^{\mathrm{a}}\end{array}$ \\
\hline 17 Sep. & 37 & Not determined & 26.6 \\
18 Sep.-24 Sep. & 102 & 53.6 & 34.1 \\
28 Sep.-29 Sep. & 75 & 100 & 82.7 \\
7 Oct. & 68 & 98.5 & 98.5 \\
19 Oct.-21 Oct. & 3 & 66.7 & 66.7 \\
23 Oct.-25 Oct. & 19 & 100 & 100 \\
31 Oct.-5 Nov. & 42 & 100 & 100 \\
16 Nov. & 7 & 100 & 100 \\
\hline
\end{tabular}

${ }^{\text {a }}$ For explanation, see Table 4.

included both mated and unmated moths.

\section{Seasonal morph}

Almost all females collected during the last week of September and later were the autumn form, and almost all females collected during the first week of October and later had fat bodies and undeveloped ovaries (Table 8). Of the females judged to be the autumn form, a few in the earlier samples had ovaries with mature eggs. This indicates that some females rated as autumn form in the earlier samples were not in reproductive diapause.

\section{DISCUSSION}

According to the data on the seasonal forms (Table 8) and a monitoring survey (Ishiguri and Kushita, unpublished), the present study covered almost two emergence periods for the 5th and 6th generations of L. p. malinella. The trough between the two generations was around 5-10 October (Ishiguri and Kushita, unpublished).
Moths flew in the morning (Table 1) and evening (Tables 2 and 3). Only males flew in the morning, whereas in the evening both males and females flew, and females in this flight included both virgin and fertilized moths (Table 6). Mating began during the morning flight period, and most copulation had finished by noon (Table 5). These findings obtained in the present study suggest that the flight in the morning is associated with mating, while that in the evening is associated with dispersal. However, further studies on earlier generations are required to make a general conclusion because the present results were derived only from the latest two generations in a year.

The swarm that flew facing the trees was different from the swarm flying close to the small branch not only in flight pattern but also in flight duration. Similar results were obtained for $P$. ringoniella (Sekita, 2002). Although much remains to be elucidated, these findings for the two species of leafminer give rise to the hypothesis that these two swarms represent different phases in a mating process. Thus, the former swarm is the flight to detect a cue such as a sex pheromone emitted from females, and the latter a direct response to the cue released by females.

Although swarming was important for locating mating partners, the present observations further showed that males were able to orient their mating partners through walking along the tree trunk. This way of locating partners is very likely to be a tactic to overcome cold weather, since it was usually observed on cold mornings. However, more research is required to establish whether its occurrence is limited to cold weather conditions.

The response of a swarm to females in traps was not only low but also unstable (Table 4). This con- 
trasted to $P$. ringoniella, where the response was high and stable, frequently attaining a full score of 4 (Sekita, 2002). This difference was reflected in the different percentages of pairing in the two species of leafminer (Table 5, and Sekita, 2002). The maximum average percentage of pairing $(=4.3 \%)$ was recorded in the middle of the morning swarming (Table 5). This was markedly lower than that for $P$. ringoniella, in which the highest percentage was over $30 \%$, and was also recorded in the middle of the morning swarming (Sekita, 2002). The difference was again reflected in the different mating status of the two species. Thus, in L. p. malinella, the proportion of females that had mated fluctuated from about 30 to $100 \%$ (Table 7), and no particular trend was noticed in these percentages during the 2-month observation period from 15 September to 16 November $(N=16$, mean $=50.5 \%$, range $=33-100 \%, r^{2}$ of a daily trend line $=0.020, p>0.1)$. In $P$. ringoniella, more than $70 \%$ of females had been fertilized at the stage when about $55 \%$ of moths had emerged in late August, and within two weeks nearly all females were fertilized (Sekita, 2002). We should bear in mind, however, that all the above serial events proceeded under warmer conditions for $P$. ringoniella than for L. p. malinella.

In these leafminer species the reproductive rates were negatively related to adult densities (Sekita, 1991). In a preliminary step to explore the underlying mechanisms of these relationships, responses of male swarms to females, percentage of pairing, and proportion of mated females should be studied in relation to population size.
Not only were females present on branches irrespective of the time of day, but males were also present there throughout the day (Tables 6 and 7). This means that some males did not fly during the times when other males were swarming. Although mating occurred during the time when males were swarming, a large proportion of males did not mate even during the prime times for mating (Table 5). All these lines of evidence suggest that males in a population are at various physiological stages. These phenomena were also the case in $P$. ringoniella (Sekita, 2002). Studies on behaviors of male moths in relation to their age would provide useful insights in this regard.

\section{ACKNOWLEDGEMENTS}

I thank my colleagues Y. Ishiguri and T. Kushita for showing me their unpublished monitoring data of L. p. malinella, and K. Yukita for drawing the map. I am also grateful to Dr. David Steven, IPM Research Ltd., New Zealand, for his generous assistance with improving my English. Further, I must acknowledge two anonymous referees for their valuable suggestions, comments and criticisms.

\section{REFERENCES}

Sekita, N. (1991) Comparative population dynamics of two species of leafminer Phyllonorycter ringoniella Matsumura and Lyonetia prunifoliella malinella Matsumura in apple orchards. Bull. Aomori Apple Exp. Stn. 27: 1-98 (in Japanese with English summary).

Sekita, N. (2002) Mass flight activity and mating in the apple leafminer Pyllonorycter ringoniella (Lepidoptera: Gracillariidae). Appl. Entomol. Zool. 37: 51-59.

Sekita, N. and M. Yamada (1979) Life history of Lyonetia prunifoliella Hübner subsp. malinella (Matsumura) (Lepidoptera: Lyonetiidae). Appl. Entomol. Zool. 14: 285-292. 